\title{
CHARGE DISTRIBUTION IN THE NITRATE ION
}

\author{
G. DE WITH* , D. FEIL \\ Chemical Physics Laboratory, Twente University of Technology, \\ Enschede, The Netherlands \\ and

\section{E.J.BAERENDS} \\ Chemical Laboratory of the Free University of Ansterdam. \\ Amsterdam, The Netherlands
}

Received 21 February 1975

The difference electron density in the nitrate ion is studied by comparison of some Hartree-Fock-Slater calculations. It is shown that good qualitative agreement with experiment is ob tained.

\section{Introduction}

Calculation of the charge distribution by means of the Hartree-Fock-Slater (HFS) model [1] seems to be very promising [2]. Recent investigation has given a further indication of the usefulness of the HFS model in electron density analysis [3]. How ever, no comparison with experimental results has been performed. For the nitrate ion experimental data are available [4] from combined $X$-ray and neutron $(X-N)$ Fourier studies of uronium nitrate. This experintental resuit is compared with double zeio and double zeta plus polarization function basis HFS calculations.

Owing to thennal smearing only a qualitative comparison with theoretical calculations can be obtained.

\section{Procedure}

Details of the (slightly idealized) geometry of the nitrate ion are given in fig. 1 . HFS wavefunctions were obtained as described in ref. [1]. For s- and ptype functions the exponents are due to Clementi [5] . The values of the exponents of the d-type functions

\footnotetext{
* To whom correspondence should be addressed.
}

are given by McLean and Yoshimine [6].

The same proceduie as in ref. [2] was used to calculate the charge distribution difference function.

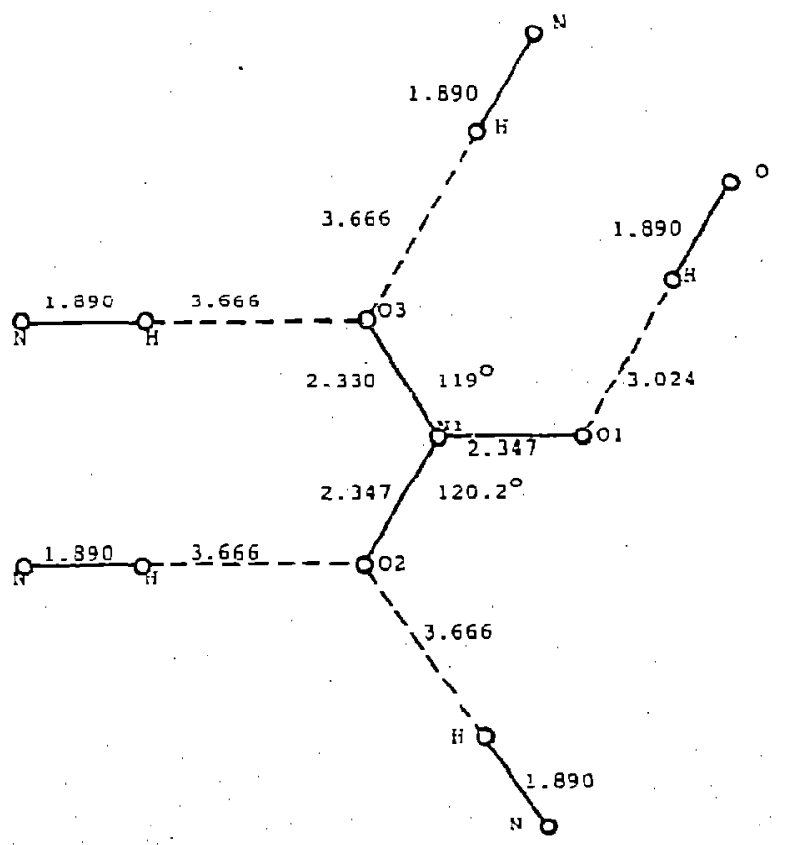

Fig. 1. Geometry of the nitrate ion as used in this work. Distance in $a_{0}$ and angles in degres. 


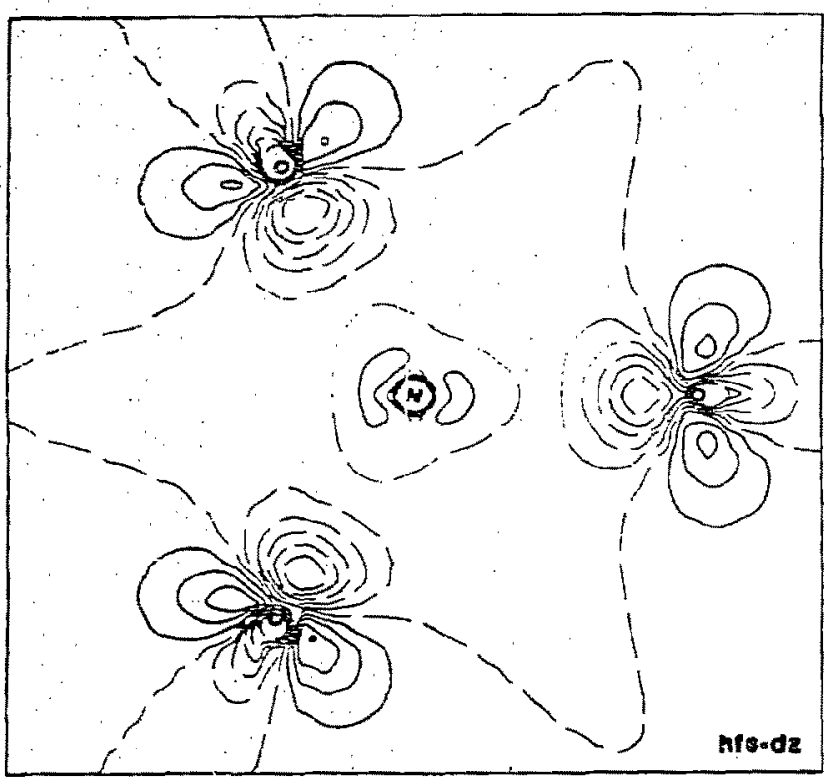

Fig. 2. Difference map for the doubic zeta basis calculation. Contours are drawn at intervals of $0.05 \mathrm{e} / \mathrm{a}_{0}^{3}$. Null and negative contours are dashed.

However, the subtracted atomic densities of oxygen were thought to have a negative charge of $\frac{1}{3} e$ each. This was reached by renormalizing the total charge contained by the p-functions of one oxygen atom to $\frac{13}{3} e$ instead of $4 e$.

\section{Results and discussion}

Results are presented for three different calculations. Fig. 2 shows the difference for a double zeta basis calculation. A few points are worth mentioning. First of all the overall height in the bonding region is very much less than the overall height in the bonding region of CO (compare ref. [2]). Secondly, the height in the lone pair lobes is about twice the height in the $\mathrm{CO}$ lone pair lobes. Furthermore, on the basis of an $\mathrm{sp}^{2}$ hybridization scheme one would expect an angle of approximately $120^{\circ}$ between the NO bond density and the lone pair electron density. A value of roughly $90^{\circ}$ is found, however. The difference map of the formate ion shows the same feature [7].

In the second calculation we considered the inclusion of poiarization functions (basis: double zeta + d-type functions on each atom). In fig. 3 we present

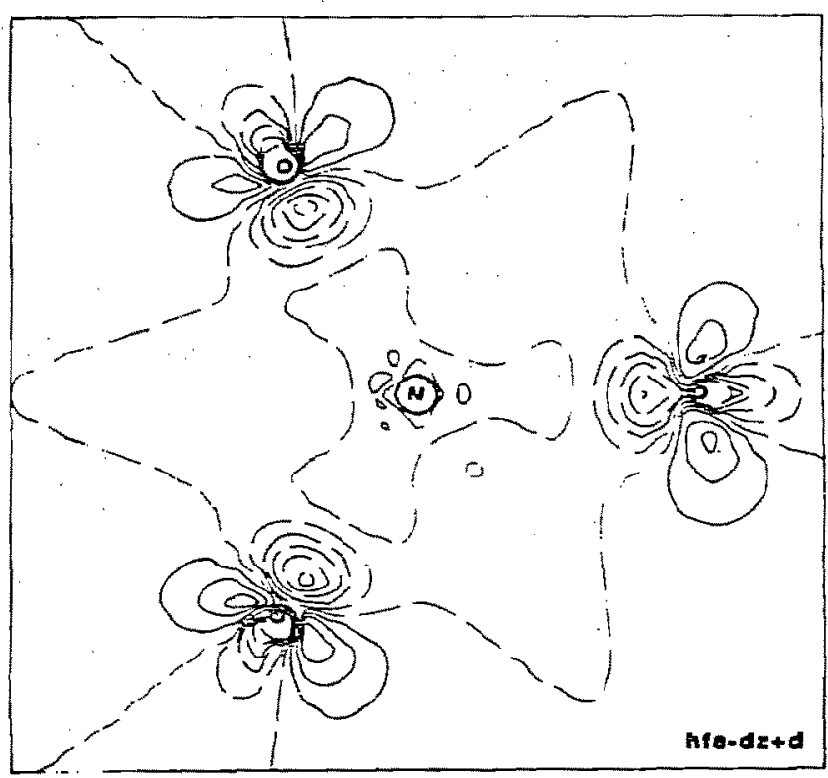

Fig. 3. Dirference map for the double zeta + d-basis. Contours are drawn similar to fig. 2 .

the difference map for this case. The electron density in the bonding region increases slightly as compared with the double zeta basis result. However, the change is not as dramatic as in the $\mathrm{CO}$ case $[2,3]$. Lone pair densities are nearly unaffected.

Finally the ion was surrounded by 5 point charges of $\frac{l}{5} e$ magnitude according to the hydrogen positions of the lydrogen bonds in the crystal structure of uronium nitrate (see fig. 2 and ref. [4] for details).

The electrostatic potential resulting from these point charges was incorporated into the Fock-operator. In this way a sort of upper limit on the effect of the environment is obtained. The second calculation showed the relative unimportance of the d-functions. Therefore the basis set was chosen of double zeta quality. The difference between the first calculation and this one is shown in fig. 4. Most interesting is the fact that there is not much difference with the normal double zeta calculation, except for the nuclear regions. This is more or less expected in view of the Iesults of Almlof et al. on $\alpha$-glycine [8]. They found a maximum difference of $0.1 e / \AA^{3}\left(\approx 0.012 e / a_{0}^{3}\right)$. However, the dependence on the crystal field is rather molecule dependent: much larger effects are estimated for the water molecule in a crystal environment $\left(0.3 e / \bar{A}^{3} \approx 0.038 e / a_{0}^{3}[7]\right)$. 


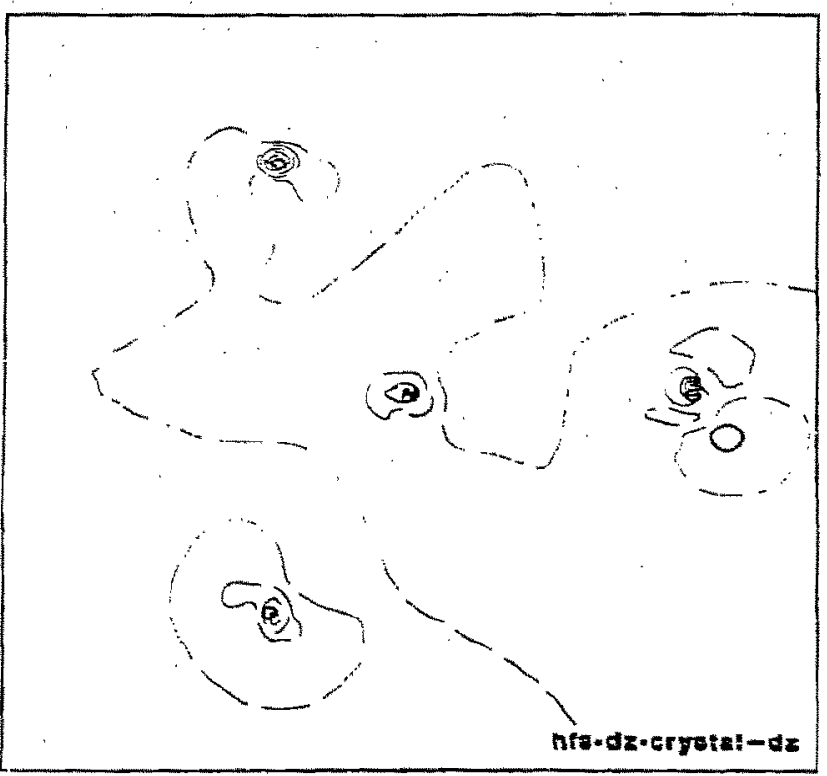

Fiv. 4. The difference between the double zeta calculation with point charges and the normal double zeta calculation. Contours are drawn at intervals of $0.005 e, a_{0}^{3}$. Null and negative contours are dashed.

For convenience the experimental difference map obtained from $\mathrm{X}-\mathrm{N}$ difference Fourier studies of uronium nitrate [4] is reproduced in fig. 5 .

Overall comparison with experiment shows a good qualitative agreement: pronounced lone pair densities and rather low or even negative bonding regions.

The direction of the lone pair lobes in the experimental map is not as extreme as in the theoretical maps. This may be partly due to librations in the plane of the ion.

\section{Summary}

Wavefunctions obtained by means of the HFS. model are rather useful in molecular charge density analysis of the nitrate ion: good qualitative agree. ment exists between experimental and theoretical difference maps. Compared with $\mathrm{CO}$ the improvement obtained by including polarization functions is less significant for $\mathrm{NO}_{3}^{-}$. The effect of the crystal field as represented by 5 point charges is negligible.

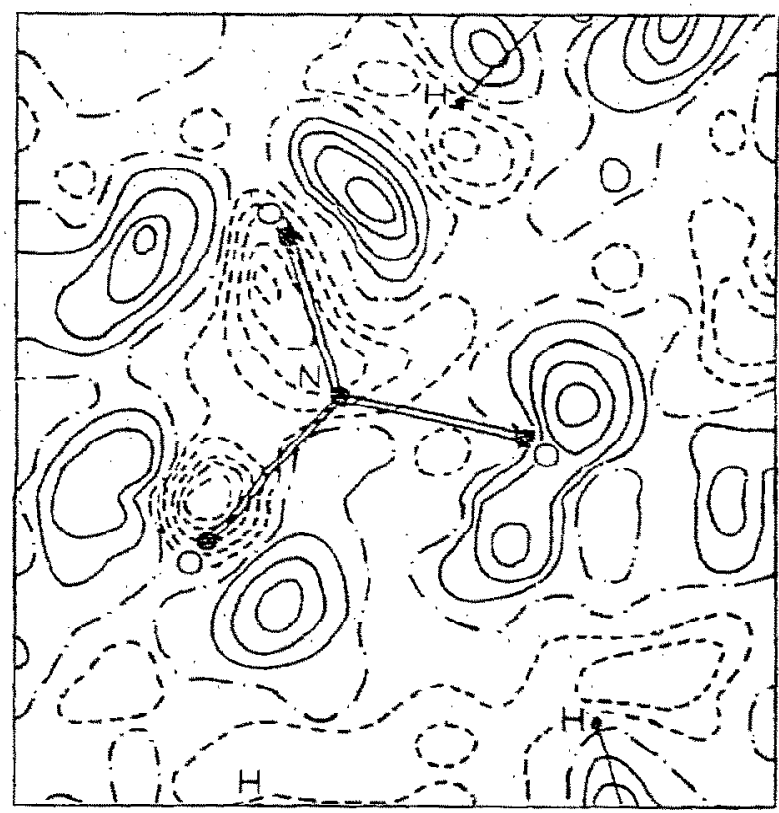

Fig. 5. Experimental difference map (taken from ref. [4]). Contours are drawn at intervals oi $0.05 \mathrm{e} / \mathrm{A}^{3}$. Null and nega" tive contours are dished.

\section{Acknowledgement}

The authors thank Dr. S. Harkema for many stim* ulating discussions. This investigation has partly been carried out under auspices of the Foundation for Fundamental Research on Matter by Electron and $X$-rays (FOMRE) and with aid from the Dutch Organization for Advancenent of Pure Research (ZWO).

\section{References}

II) E.J. Baerends, D. Ellis and P. Ros, Chem. Phys: 2 (1973) 1.

[2] G. de With and D. Fei, Chem. Phys. Letters 30 (1975) 279.

(3) G. de With and E. Bacrends, unpublished results.

[4] S. Harkema, thesis, Twente University of Technology, Enschede, The Netherlands.

(5) E. Clementi, IBM I: Res. Develop. 9 (1965) 2, suppl.

(6) A.D. McLean and M. Yoshimine, IBM J. Res, Develop, 11 (1967), suppl.

(7) 1 . Almlöf, private communtcation.

[8] I. Almlö, A. Kvick and 1,O. Thomas, J. Chem Phys 59 (1973) 3901 